\title{
High-pressure treatment reduces the immunoreactivity of the major allergens in apple and celeriac
}

\author{
Fiona A. Husband ${ }^{1}$, Thomas Aldick ${ }^{1}$, lesel Van der Plancken ${ }^{2}$, Tara Grauwet ${ }^{2}$, \\ Marc Hendrickx ${ }^{2}$, Isabel Skypala ${ }^{3}$ and Alan R. Mackie ${ }^{1}$ \\ ${ }^{1}$ Structuring Food for Health Programme, Institute of Food Research, Norwich Research Park, Colney, UK \\ ${ }^{2}$ Laboratory of Food Technology and Leuven Food Science and Nutrition Research Centre (LFoRCe), Department of \\ Microbial and Molecular Systems (M2S), Katholieke Universiteit Leuven, Leuven, Belgium \\ ${ }^{3}$ Nutrition and Dietetics Department, Royal Brompton Hospital, London, UK
}

Scope: The impact of thermal and high pressure (HP) processing on the immunoreactivity of the allergens Mal d 1, Mal d 3 and Api g 1 has been investigated in apple and celeriac tissue, respectively.

Methods and results: The extracted proteins were assessed using SDS-PAGE and Western blot. The results showed that Mal d 1 was subject to loss of immunoreactivity as soon as the apple tissue was disrupted although it was remarkably resistant to both thermal and HP processing. This is in contrast to the Mal d 1 structural homolog from celeriac, Api g 1, that was susceptible to thermal processing. The other major allergen in apple, Mal d 3, was found to be resistant to chemical modification and thermal processing in apple, which is in contrast to behavior in solution. However, the combination of pressure and temperature significantly reduced its immunoreactivity. Pectin was found to protect Mal d 3 from thermal denaturation in solution and is a possible candidate for the protective effect of the fruit.

Conclusion: The conclusion to be drawn from these results is that the combination of HP and thermal processing is an effective method to reduce the allergenicity of both apple and celeriac.
Received: November 12, 2010

Revised: February 1, 2011

Accepted: February 18, 2011
Keywords:

Allergy / Apple / Celeriac / High pressure / Processing

\section{Introduction}

A combination of high pressure (HP) and mild thermal treatments are considered novel processing techniques that have the potential to alleviate the need for more severe thermal processing of foods. HP (400-700 MPa) combined with temperatures around room temperature $\left(20-40^{\circ} \mathrm{C}\right)$ can be used to pasteurize foods [1-3]. For example, a HP pasteurization process of $500 \mathrm{MPa}$ for $10 \mathrm{~min}$ was capable of inactivating more than $5 \mathrm{log}$ decades of the viable

Correspondence: Dr. Alan R. Mackie, Structuring Food for Health Programme, Institute of Food Research, Norwich Research Park, Colney, NR4 7UA, UK

E-mail: alan.mackie@bbsrc.ac.uk

Fax: +44-1603-507723

Abbreviations: HP, high pressure; HP/T, high pressure/thermal; LTP, lipid transfer protein; PBST, phosphate-buffered saline with Tween 20; TBST, Tris buffered saline/Tween microorganisms present originally in a raw apple/broccoli juice [1]. These treatments offer an alternative to conventional high-temperature pasteurization, or chemical preservation because they are less destructive of factors associated with fresh-like character in foods and as a result have been investigated extensively [4-7]. It is the potential of HP processing to act as an alternative method of preventing food spoiling while retaining physically desirable characteristics in foods that makes it such an appealing prospect for the future. For example, HP/T (high pressure/thermal) processing has been used in the manufacture of rice-based products such as cooked rice, rice crackers and rice cakes [8]. The effect of HP and/or thermal treatment on proteins, and in particular enzymes, has also been widely studied [5, 9-11]. The effect of thermal treatment at HP on pectin has been shown to improve cell wall strength over treatment at ambient pressure by decreasing $\beta$-elimination and increasing the rate of demethoxylation [5]. In another study looking at mustard seed myrosinase [10], it was found that at 
atmospheric pressure, the optimal temperature for activity in Bis-Tris buffer at $\mathrm{pH} 6.5$ was $60^{\circ} \mathrm{C}$ while at elevated pressure, the reaction rate increased until $200 \mathrm{MPa}$ and the optimal temperature shifted to $40^{\circ} \mathrm{C}$. In broccoli juice, the reaction rate also increased up to pressures of $200 \mathrm{MPa}$, as was found in buffer solution. However, the myrosinase reaction rate in broccoli juice was higher still, at $400 \mathrm{MPa}$. HP treatment has also been seen as a method of reducing the allergenic potential of foods such as apple [12], beef [13] and egg [14]. Additionally, the work of Asero et al. has shown that the lipid transfer protein (LTP) from apple (the allergen Mal d 3) is extremely heat stable [15].

Apple is one of the most common allergenic foods in Europe and contains two predominant allergens, Mal d 1 and Mal d 3. Apple Mal d 3 is a non-specific lipid transfer protein, a member of the prolamin structural family [16] and is a problematic allergen in Southern European populations [17]. The second allergen, Mal d 1, is a member of the PR-10 structural family. It is allergenic because of cross-reactivity with another member of the same structural family, Bet $\mathrm{v} 1$ from Birch pollen. Mal d 1 is a major allergen in Northern European populations and is implicated in pollen associated fruit allergy [17]. Mal d 1 is a member of a large gene family of Bet $\mathrm{v} 1$ related plant proteins, the major expressed fruit form of the protein being Mal d 1b [18]. This lab has previously studied the effect of HP processing and temperature on purified Mal d 1 and Mal d 3 from apple [19]. Pressure treatment alone produced very little change in secondary structure in the purified protein. Secondary structure was changed significantly when pressure/temperature combinations were used. The immunoreactivity of the allergens correlated well with the decline in secondary structure as observed by circular dichroism spectroscopy.

While in the UK celery stem is mainly consumed, celery root or celeriac is widely consumed in central Europe and often in a processed form such as celery salt that is made from a combination of salt and either celery seeds or extract of celeriac. Previous studies by Jankiewicz et al. using a combination of polyclonal antibodies and human allergic sera have shown the effect of various types of processing [20]. They showed that thermal processing (microwave to $>100^{\circ} \mathrm{C}$ ) for $10 \mathrm{~min}$ reduced the reactivity to polyclonal antibodies raised against Bet $\mathrm{v} 1$ and that microwave heating for $30 \mathrm{~min}$ completely removed the reactivity. In addition, they showed that $\mathrm{HP}$ processing at $600 \mathrm{MPa}$ at $20^{\circ} \mathrm{C}$ significantly reduced the polyclonal reactivity of Api g 1 . In vivo studies involving food challenges in celeriac allergic patients have also shown that the PR 10 allergen in celeriac, Api g 1 , was very much reduced in its allergenicity by thermal processing $\left(110^{\circ} \mathrm{C}\right.$ for $15 \mathrm{~min}$ [21]. These results suggest HP and thermal processing can decrease the allergic potential of Api g 1 in celeriac.

Thermal processing at temperatures in excess of $80^{\circ} \mathrm{C}$, of the purified PR 10 allergens from plants that cause pollen associated allergy, such as apple or celery has been shown to decrease their IgE reactivity [22]. However, this may not be representative of what happens when the allergens are heated in the fruit, where the chemical environment of the proteins may be very different. The aim of this paper is to assess whether the effects of HP treatment of apple tissue reduces the immunoreactivity of the primary allergens contained within it.

\section{Materials and methods}

\subsection{Apple peel preparation and treatment}

\subsubsection{Preparation}

Apples (Fuji) were purchased from a local market and stored for up to $2 \mathrm{wk}$ at $4{ }^{\circ} \mathrm{C}$ prior to use. Before treatment, they were equilibrated at room temperature. Apples were peeled (1-2 mm thickness with a household apple peeler) and the peel was vacuum sealed in plastic bags (within $7 \mathrm{~min}$ ). No additional steps were taken to prevent oxidation. Each sample comprised the skin of a quarter of an apple. Two samples and two references were made from each apple, and this was done in duplicate to repeat each treatment condition.

\subsubsection{HP treatment}

HP experiments were performed in a laboratory-scale multivessel HP equipment (Resato, Roden, The Netherlands), consisting of six individual vessels surrounded by an isolated heating coil, connected to a cryostat. This equipment allows computer-controlled pressure build-up, temperature control to temperatures up to $120^{\circ} \mathrm{C}$ and data logging of both pressure and temperature.

Pressure treatment was performed within $15 \mathrm{~min}$ of peeling the apple. Reference samples were stored at room temperature during the pressure treatment of the corresponding samples. The vacuum-sealed bags containing apple peel were transferred to a water-filled sample holder at room temperature. The sample holders were inserted into the thermally equilibrated vessels (at 20 or $115^{\circ} \mathrm{C}$ ) within $2 \mathrm{~min}$. Temperature was monitored with a thermocouple inserted in one of the sample holders (sample not used for analysis). After a pre-determined time, during which the sample heated up to the initial temperature $T_{\mathrm{i}}$ (dependent on the target pressure) the pressure was increased to $700 \mathrm{MPa}$ (first immediately to $150 \mathrm{MPa}$, then at $10 \mathrm{MPa} / \mathrm{s}$ ). The pressure vessels were allowed to equilibrate for $2 \mathrm{~min}$ at the target pressure before the hold time of $10 \mathrm{~min}$. After the hold time, the pressure was completely released. The samples were removed from the HP equipment within $1 \mathrm{~min}$ and immediately transferred to an ice bath to stop any further changes. All samples including controls were frozen in liquid nitrogen and stored at $-80^{\circ} \mathrm{C}$ prior to analysis. The temperature-pressure profile of this treatment is shown in Fig. 1. In all samples except those HP treated over $600 \mathrm{MPa}$, browning was very evident and in all samples the firmness had been reduced to a rubbery texture. 


\subsubsection{Thermal treatment}

Thermal treatment at $115^{\circ} \mathrm{C}$ at ambient pressure was performed within $15 \mathrm{~min}$ of peeling the apple. Reference samples were stored at room temperature during the thermal treatment of the corresponding samples. The vacuumsealed bags containing apple peel were transferred to a water-filled sample holder at room temperature. Temperature was monitored with a thermocouple inserted in one of the tubes (sample not used for analysis). After attaining the process temperature (within $2^{\circ} \mathrm{C}$ ), a holding time of $10 \mathrm{~min}$ was applied. After this holding time, the tubes were transferred to an ice water bath. Samples were frozen in liquid nitrogen and stored at $-80^{\circ} \mathrm{C}$ prior to analysis.

In addition, we also prepared a number of experiments that represented the traditional oven baking of an apple. In these, an apple was placed in an oven at $180^{\circ} \mathrm{C}$ and cooked for $30 \mathrm{~min}$. After $30 \mathrm{~min}$ at $180^{\circ} \mathrm{C}$, the temperature of the core area of the apple was determined to be $92^{\circ} \mathrm{C}$ by a thermocouple. The baked apple was then removed from the incubator and allowed to cool for 15-20 min before skin and flesh samples were removed. These samples were frozen on dry ice and used immediately.

\subsection{Celeriac cylinder preparation and treatment}

\subsubsection{Preparation}

Celeriac root was purchased from a local market and stored for up to $2 \mathrm{wk}$ at $4^{\circ} \mathrm{C}$ prior to use. Before treatment, they were equilibrated at room temperature. Cylinders $(2 \mathrm{~cm}$ diameter in case of pressure treatment, $1.2 \mathrm{~cm}$ in case of thermal treatment) were drilled. For pressure treatment, about $9 \mathrm{~cm}$ of celeriac cylinder was vacuum sealed in a plastic bag (two layers) for each sample (within $7 \mathrm{~min}$ ), while for thermal treatment, about $15 \mathrm{~cm}$ cylinders were vacuum sealed in a plastic bag (one layer). Four samples and four references were made from half of a celeriac, and this was done in duplicate to repeat each processing condition.

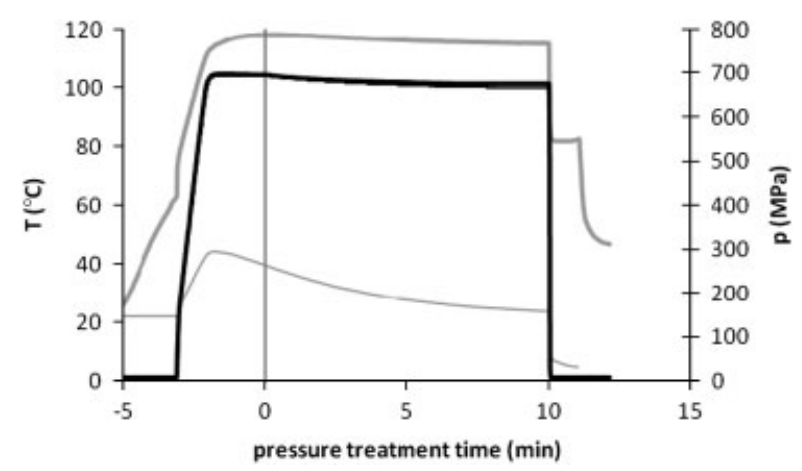

Figure 1. Temperature (gray) and pressure (black) profiles monitored in sample holders containing vacuum-sealed apple peel, pressure treated at $20^{\circ} \mathrm{C}$ (thin line) or $115^{\circ} \mathrm{C}$ (thick line) and $700 \mathrm{MPa}$.

\subsubsection{HP treatment}

The vacuum-sealed bags containing the celeriac cylinders were directly (not in a sample holder) inserted in the thermally equilibrated vessels (at 20 or $118^{\circ} \mathrm{C}$ ) within $2 \mathrm{~min}$. The remainder of the procedure was as described for the apple peel.

\subsubsection{Thermal treatment}

The bagged celeriac cylinders were hermetically closed into water-filled stainless steel tubes with a headspace of $1 \mathrm{~cm}$. Celeriac cylinders were treated in these tubes in a thermostatted oil-bath $\left(118^{\circ} \mathrm{C}\right)$. The remainder of the procedure was as described for the apple peel (Table 1).

\subsection{Purified Mal d 1b/apple pectin mixtures}

Purified recombinant Mal d 1b was prepared according to Johnson et al. [19] and in combination with apple pectin (Sigma, Poole, UK. Cat. No. 76282, 70-75\% esterification) was heated to $105^{\circ} \mathrm{C}$ for $10 \mathrm{~min}$, in $10 \mathrm{mM}$ phosphate buffer, $\mathrm{pH}$ 7.0. Samples were used at protein to pectin weight ratios of 1:10, 1:1 and 10:1 and at a standard concentration of $0.25 \mathrm{mg} / \mathrm{mL}$ protein. Following heating, the samples were cooled and then used for SDS-PAGE electrophoresis (using $100 \mu \mathrm{L}$ sample $+40 \mu \mathrm{L}$ sample buffer $+15 \mu \mathrm{L} \quad$ DTT) as described in Section 2.4.1.

\subsection{Analysis}

\subsubsection{SDS-PAGE}

Frozen apple peel was ground with dry ice using a coffee grinder (Kinematica, Switzerland) until it resembled a frozen fine powder (approximately $2 \times 15 \mathrm{~s}$ ). Samples of celeriac were taken from more than $0.5 \mathrm{~cm}$ away from the outer skin. The samples were ground with dry ice in a coffee grinder

Table 1. Pressure and temperature conditions used to treat the apple and celeriac samples

\begin{tabular}{llll}
\hline Code & Temperature $\left({ }^{\circ} \mathrm{C}\right)$ & Pressure $(\mathrm{MPa})$ & Time $(\mathrm{min})$ \\
\hline A & Ambient & Ambient & 1 \\
A1 & 20 & Ambient & 10 \\
B & 20 & Ambient & 60 \\
C & 50 & Ambient & 60 \\
D & 180 & Ambient & 30 \\
E & 115 & Ambient & 10 \\
E1 & 118 & Ambient & 10 \\
F & 20 & 700 & 10 \\
G & 115 & 700 & 10 \\
G1 & 118 & 700 & 10 \\
H & 105 & Ambient & 10 \\
\hline
\end{tabular}


$(2 \times 15 \mathrm{~s})$. An aliquot $(0.15 \mathrm{~g})$ of frozen apple/celeriac powder was added to $200 \mu \mathrm{L}$ NuPage LDS sample buffer (Invitrogen, Cat. No. NP0007) and $100 \mu \mathrm{L}$ DTT $(77 \mathrm{mg} / \mathrm{mL})$; the samples were heated to $70^{\circ} \mathrm{C}$ for $10 \mathrm{~min}$ prior to loading onto SDSPAGE. Four to twelve percent Bis-Tris gels (Invitrogen, Cat No. NP0322BOX) were used with MES running buffer (Invitrogen, Cat No.NP0002). Gels were run for $35 \mathrm{~min}$ at $200 \mathrm{~V}$ and stained using the Novex Colloidal blue staining kit (Invitrogen, cat No. LC6025). Molecular weight markers used were in the standard set SeeBlue Plus 2 (Invitrogen, Cat. No LC5925) for the gels and in the standard set RunBlue (Expedeon, Cat. No. NXA05160) for the Western blots.

\subsubsection{Antibodies}

Mal d 3 antibodies were raised in rabbit at IFR. A solution of Mal d 3 purified from apple peel using the method of Sancho et al. [23] $(0.25 \mathrm{~mL}$ of $0.4 \mathrm{mg} / \mathrm{mL}$ in sterile saline solution, $0.85 \% \mathrm{NaCl}$ ) was emulsified with $0.5 \mathrm{~mL}$ of TiterMax Gold adjuvant (Sigma, Dorset, UK) by repeatedly passing the mixture through a narrow aperture between syringes. Half the emulsified protein was used to inoculate each of two male rabbits (New Zealand White). The inoculations were repeated twice at monthly intervals after the initial inoculation. Blood was collected 15 days after the final booster injection from ear bleeds into heparinized tubes and centrifuged at $720 \times \mathrm{g}$ for $15 \mathrm{~min}$ at room temperature to remove cells. The serum fraction was then removed and stored frozen in aliquots at $-20^{\circ} \mathrm{C}$. Serum from the animal that gave the highest titer against Mal d 3 was selected for further work in the study. The polyclonal antibodies to natural Bet $\mathrm{v} 1$ were raised in New Zealand White rabbits as described in Zuidmeer et al. [24]. These were used to detect both Mal d 1 and Api g 1. The secondary antibody used was goat anti-rabbit conjugated to alkaline phosphatase (Sigma, UK, Cat. No. A8025).

\subsubsection{Human Sera}

Ethical permission had been granted for the collection and subsequent usage of the human sera, as part of the ISAfruit study (www.isafruit.org). The ISAfruit study samples were collected through The Royal Brompton and Harefield NHS Trust. A pool of human sera, comprising five individual sera, were used. These sera were selected for their high individual reactivity; the mixed pool would have a titer of approx 1:100. The self-reported reaction history and skin prick (prick to prick against positive and negative controls) test results of the patients are given in Table 2.

\subsubsection{Immunoblots}

Electrophoresis was run as described above. The gel was then sandwiched between blot paper (BioRad, Cat. No.

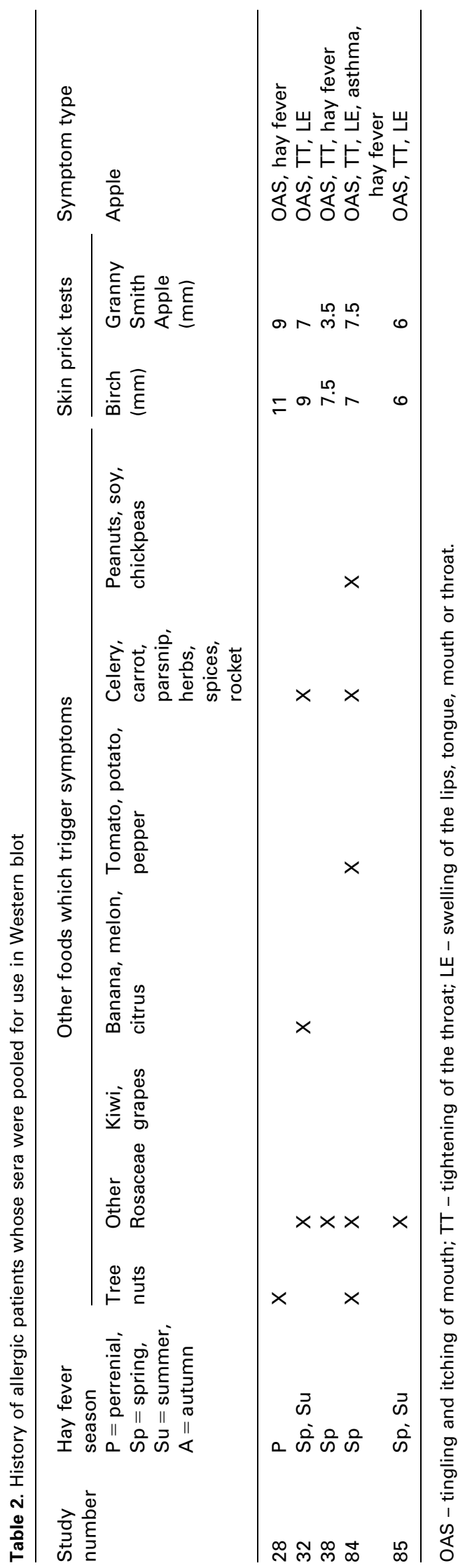

(c) 2011 WILEY-VCH Verlag GmbH \& Co. KGaA, Weinheim 
1703965) with nitrocellulose. The sandwich was then placed in a Bio-Rad Transblot-SD Semi-dry transfer cell and blotted for $20 \mathrm{~min}$ at $15 \mathrm{~V}$. The nitrocellulose was incubated with phosphate-buffered saline containing Tween 20 (PBST) containing 5\% skimmed milk powder for $1 \mathrm{~h}$ at room temperature with shaking. The nitrocellulose was washed three times with PBST prior to incubating with the primary antibody $(1: 10000 \mathrm{v} / \mathrm{v}$ in PBST) for $1 \mathrm{~h}$ at room temperature with shaking. The nitrocellulose was washed a further three times, prior to incubating with the secondary antibody $(1: 1000 \mathrm{v} / \mathrm{v}$ in PBST) for $1 \mathrm{~h}$ at room temperature with shaking. A further washing step with PBST followed by distilled water was used before developing using 5-bromo-4chloro-3-indolyl phosphate/Nitro blue tetrazolium (BCIP/ NBT) (Sigma, Cat. No.B5655).

\subsubsection{IgE blot development}

Samples were prepared for electrophoresis and blotting as described above. The blot was then incubated with $30 \mathrm{~mL}$ Tris-buffered saline/Tween (TBST: $0.05 \mathrm{M}$ Tris, $0.138 \mathrm{M}$ $\mathrm{NaCl}, 0.0027 \mathrm{M} \mathrm{KCl}, 0.05 \% \mathrm{v} / \mathrm{v}$ Tween 20; $\mathrm{pH} 8.0$ ) containing $5 \% \mathrm{w} / \mathrm{v}$ skimmed milk powder for $1 \mathrm{~h}$ with gentle agitation. The blot was then washed three times with TBST and incubated with diluted human sera (1:10), at room temperature, overnight. The blot was drained and washed three times with TBST and incubated with goat anti-human IgE-alkaline phosphatase conjugate (Pharmingen, 555859) (1:3500 in TBST) for $2 \mathrm{~h}$. After a final wash with TBST, it was stained with 5-bromo-4-chloro-3-indolyl phosphate/ Nitro blue tetrazolium as above.

\subsubsection{Densitometry}

The gels were scanned in a BioRad GS800 Calibrated densitometer at resolution of $300 \mathrm{dpi}$. Line profiles of the middle $50 \%$ of the bands were examined. The maximum peak heights of reflective density minus the background values were then quoted as a percentage of the result obtained for the fresh sample, allowing easier comparison between apple and celeriac.

\section{Results}

\subsection{Modifications in allergens during thermal treatment}

To understand the impact of low levels of thermal processing of apple on the allergens contained within it, a number of heating experiments were undertaken. Samples of apple were treated and frozen. The protein was then extracted from the frozen samples using the methods outlined above. Initially, a comparison was made between fresh samples and those incubated for $1 \mathrm{~h}$ either at 20 or $50^{\circ} \mathrm{C}$, treatments A, B and C, respectively. The results (Fig. 2) reveal the presence and detection of Mal d 3 (using a Mal d 3 antibody) and Mal d 1 (using an anti-Bet v 1 antibody) following thermal processing of the powdered apple tissue. As a comparison, another Bet v 1 homologue, Api g 1, from celeriac was also treated and detected in the Western blot using the same anti-Bet $\mathrm{v} 1$ antibody as used for detection of Mal d 1. The data from Fig. 2A contain a large number of bands corresponding to the large number of molecules of different sizes extracted from the apple. The data in Fig. 2B that primarily show the bands of the Mal d 3 (lanes 1-3) Mal d 1 (lanes 4-6) and Api g 1 (lanes 7-9) are much clearer, allowing the band density to be measured.

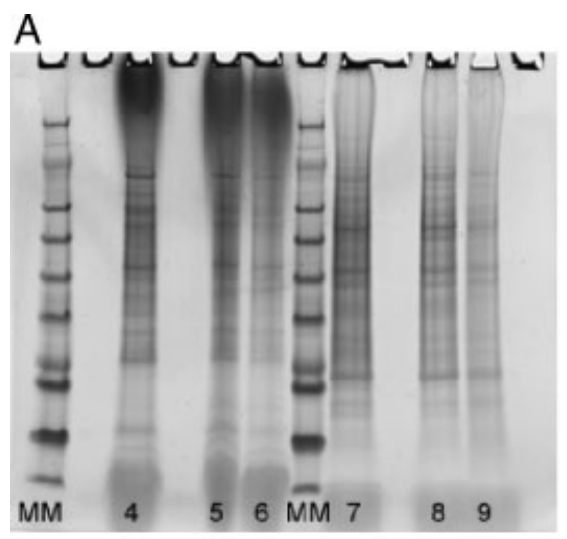

B

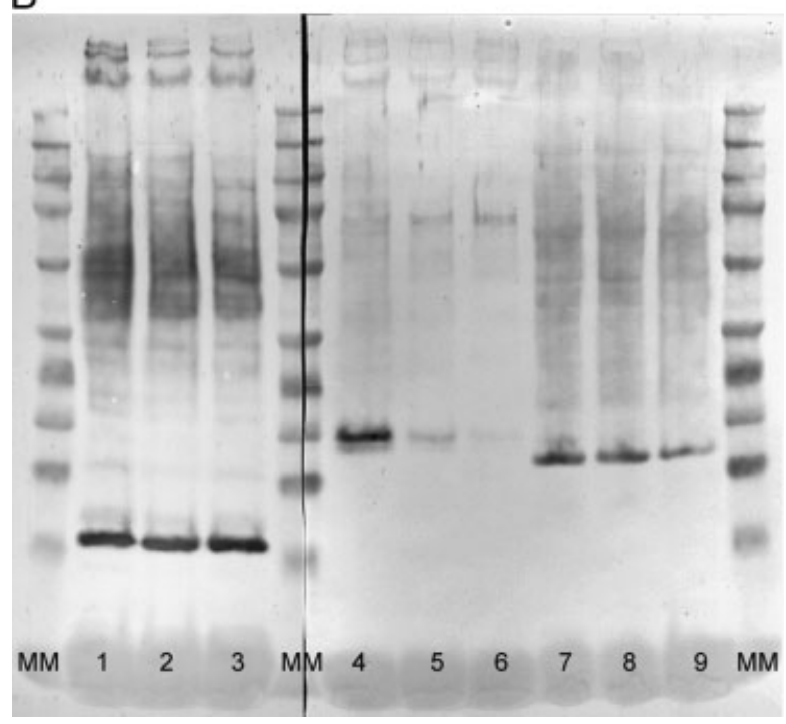

Figure 2. (A) SDS PAGE gel and (B) Western blot detecting Mal d 3 (lanes 1-3), Mal d 1 (lanes 4-6) and Api g 1 (lanes 7-9). The samples comprised apple treatment $A$ (lanes 1 and 4), apple treatment $B$ (lanes 2 and 5 ) and apple treatment $C$ (lanes 3 and 6 ). Lanes 7-9 are celeriac treatment $A, B$ and $C$, respectively. Molecular weight standards are run in lanes marked MM. Lane numbers in the gel and blot correspond to the same samples. 
To follow the effect of heating the protein while still in the fruit, apples were put through a typical baking process (treatment D). Figure 3A lanes 1 and 2 show that Mal d 3 was exclusively found in the apple peel and not in the cortex as seen previously [25]. Lane 3 also shows that it survived in the skin of an apple that underwent a typical baking process. The blot for Mal d 1 in Fig. 3B shows that while most of the Mal $d 1$ was in the peel sample, there was also protein in the cortex sample, lanes 1 and 2, respectively. After heating, however, there was essentially no difference in the amount of immunoreactive Mal d 1 between the peel and cortex samples.

As Mal d 3 has been associated with apple cell wall [26], the potential protective effect of pectin on the thermal denaturation of Mal d 3 in solution was also investigated. As previously shown, heating Mal d 3 in solution to tempera-

\section{A}

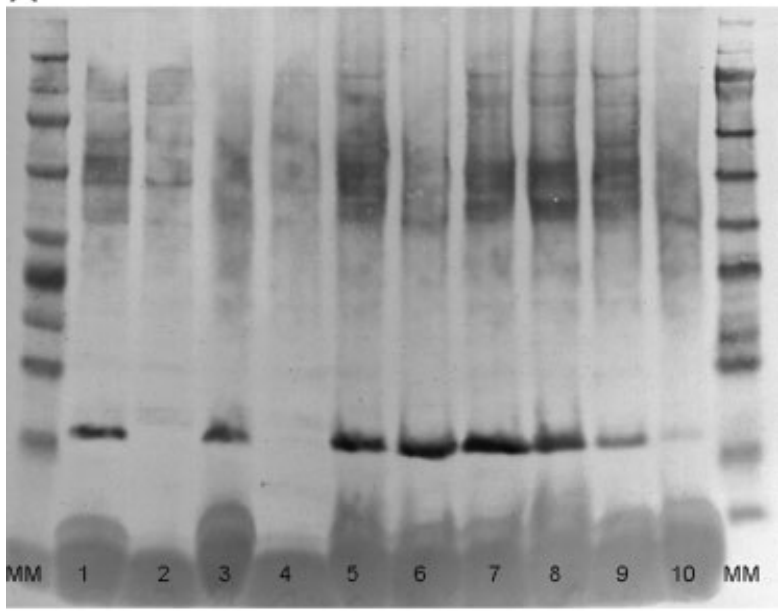

B

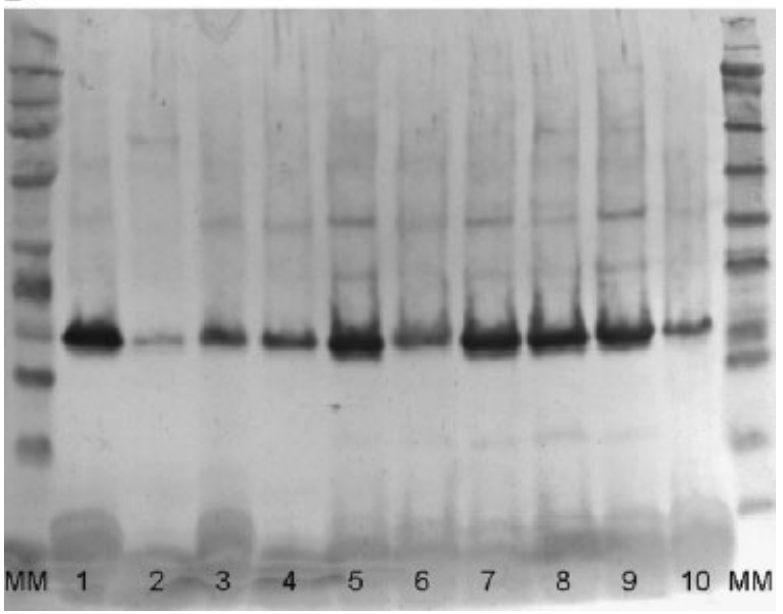

Figure 3. Western blots to (A) Mal d 3 and (B) Mal d 1. The samples are as follows: 1 - control peel; 2 - control cortex; 3 - peel treatment $D ; 4-$ cortex treatment $D ; 5$ - control apple; 6 - apple treatment $\mathrm{E} ; 7$ - control apple; 8 - apple treatment $\mathrm{F}$; 9 - control apple; 10 - apple treatment G. Molecular weight markers are labeled MM. tures above $100^{\circ} \mathrm{C}$ caused the protein structure to be modified. This is illustrated by broadening of the protein band in Fig. 4A, track 2 and the decrease in intensity of the same band in Fig. 4B. However, heating in the presence of pectin at ratios as low as 10:1, protein/pectin significantly protected the protein from modification as shown in particular by the clarity of the bands in tracks 6,8 and 10 in Fig. 4A. This shows that while the pectin may not be the only compound limiting the denaturing of Mal d 3, it has the capacity to play a role.

A

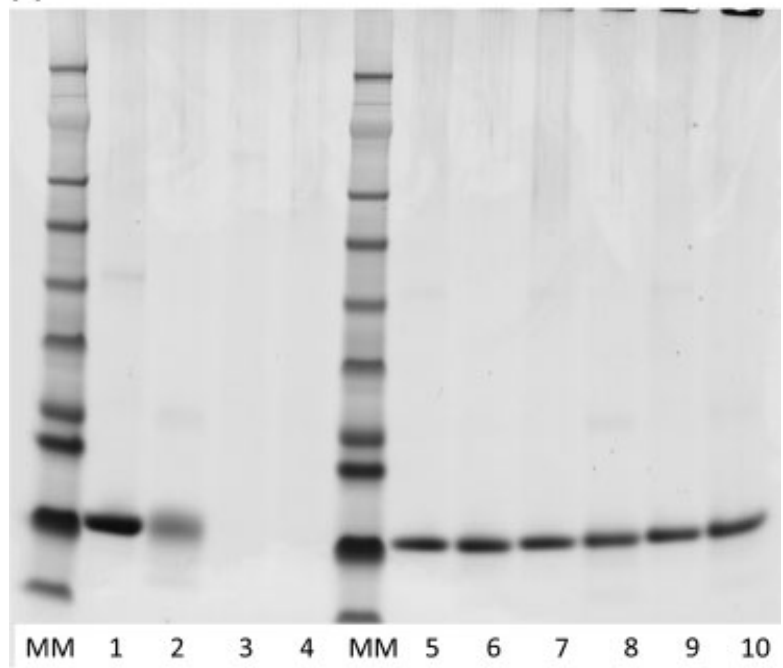

$\mathrm{B}$

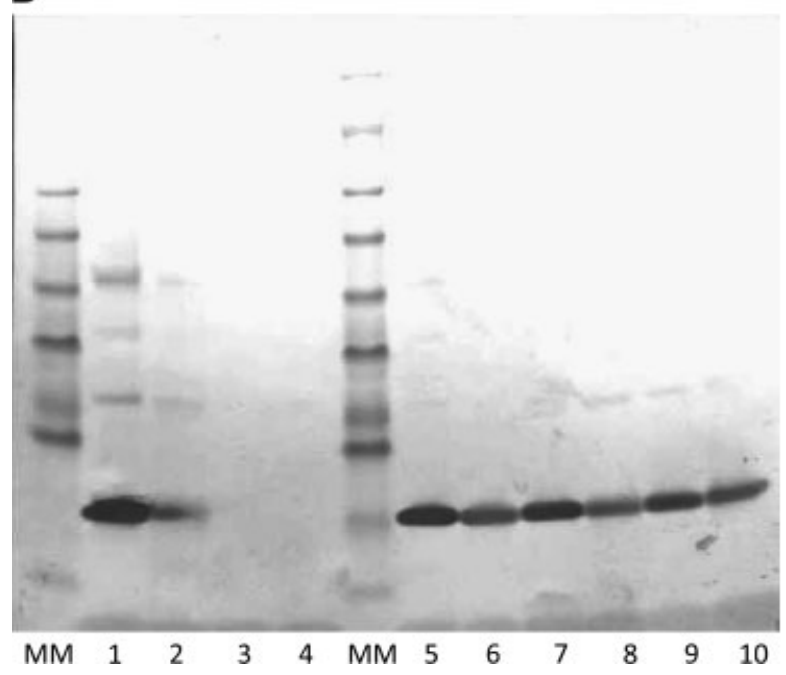

Figure 4. Effect of pectin on the thermal modification of Mal d 3 as detected by (A) reducing SDS-PAGE and (B) immunoblot. Samples are as follows: 1 - Mal d 3 unheated; $2-$ Mal d 3, $105^{\circ} \mathrm{C}$, $10 \mathrm{~min} ; 3$ - apple pectin; 4 - apple pectin, $105^{\circ} \mathrm{C}, 10 \mathrm{~min} ; 5$ - Mal d 3/pectin 10:1 (w/w) unheated; 6 - Mal d 3/pectin 10:1 (w/w), $105^{\circ} \mathrm{C}, 10 \mathrm{~min} ; 7-$ Mal d 3/pectin 1:1 (w/w) unheated; 8 - Mal d 3/pectin 1:1 (w/w), $105^{\circ} \mathrm{C}, 10 \mathrm{~min} ; 9-$ Mal d 3/pectin 1:10 (w/w) unheated; 10 - Mal d 3/pectin 1:10 (w/w), $105^{\circ} \mathrm{C}, 10 \mathrm{~min}$. 


\subsection{Modifications in allergens during pressure treatment}

The results from the thermal processing were confirmed and any additional changes induced by HP (either at ambient or elevated temperature) were assessed by a series of HP treatments undertaken on apple peel. The results of Western Blot analysis of the extracted samples are also shown in Fig. 3. Lanes 5-10 in Fig. 3A show the affect of heating and HP on Mal d 3 immunoreactivity. Lane 6 shows that thermal treatment alone (treatment E) did not change the amount of immunoreactive Mal d 3 present in comparison to the control sample in lane 5. HP processing (treatment F) caused a slight decrease ( $70 \%$ by densitometry) in the amount of immunoreactive Mal d 3 (lane 8 compared to lane 7). Mal d 3 still remains in lane 10 (treatment $\mathrm{G}$ ) compared to the control sample in lane 9. The impact of HP/T processing on Mal d 1 as shown in Fig. 3B was somewhat less marked than the effect on Mal d 3. Lane 6 shows that treatment E decreased the amount of immunoreactive protein by about $40 \%$, but the HP processing in treatment $\mathrm{F}$ had no effect on the protein. Surprisingly, even the most extreme conditions only reduced the amount of immunoreactive protein by a little over $50 \%$.

As a comparison with the effects of $\mathrm{HP} / \mathrm{T}$ on apple allergen Mal d 1, samples of celeriac were treated under the same conditions and both SDS PAGE and Western blots were made. The results are shown in Fig. 5 and reveal slightly different behavior to that of the apple. As in Fig. 2A, the SDS PAGE in Fig. 5A shows many bands from molecules extracted from the celeriac including a band of a molecular weight indicating the presence of Api g 1 . However, the blot in Fig. 5B shows only single bands for the two samples treated at $20^{\circ} \mathrm{C}$ in lanes 1 and 2 and a very faint band in lane 4.

Having detected changes in immunoreactivity with polyclonal antibodies, it was necessary to assess whether the changes seen in Mal d 1 as a result of pressure and temperature were representative of the likely reactivity in human apple allergic patients; IgE blots were undertaken using a pool of human sera as described above. The resulting blot and densitometry data are shown in Fig. 6. As in Fig. 3B, the blot in Fig. 6A lanes 1 and 2 shows a marked decrease in reactive Mal d 1 after treatment $E$ but a much smaller decrease after pressure treatment F, lanes 3 and 4. Not surprisingly there was also a marked decrease in reactive Mal d 1 after pressure treatment $G$, lanes 5 and 6 . Figure 6B compares the relative densities of all the blot data. Both the data for IgG and IgE show similar trends, with the primary sensitivity being to thermal processing rather than HP and in all cases the IgE was more sensitive to the structural changes induced by combination of thermal and HP processing.

\section{Discussion}

We have assessed the effect of HP and thermal processing on the allergens in apple. Regardless of any sort of proces- sing, we have shown in Fig. 2 that the immunoreactivity of Mal d 1 diminished after the apple flesh was cut. Additionally, the rate at which the immunoreactivity of Mal d 1 diminished increased at the elevated temperature of $50^{\circ} \mathrm{C}$. This was probably due to chemical modification of the protein by enzymes in the apple. A likely candidate enzyme is polyphenol oxidase, which has already been shown to reduce the IgE reactivity of Mal d $1[27,28]$. This is in contrast to the Api g 1 treated in celeriac, which showed only a limited decrease in reactivity compared to the apple samples. It is not possible to say that this is because of differences in PPO concentrations in apple and celeriac as

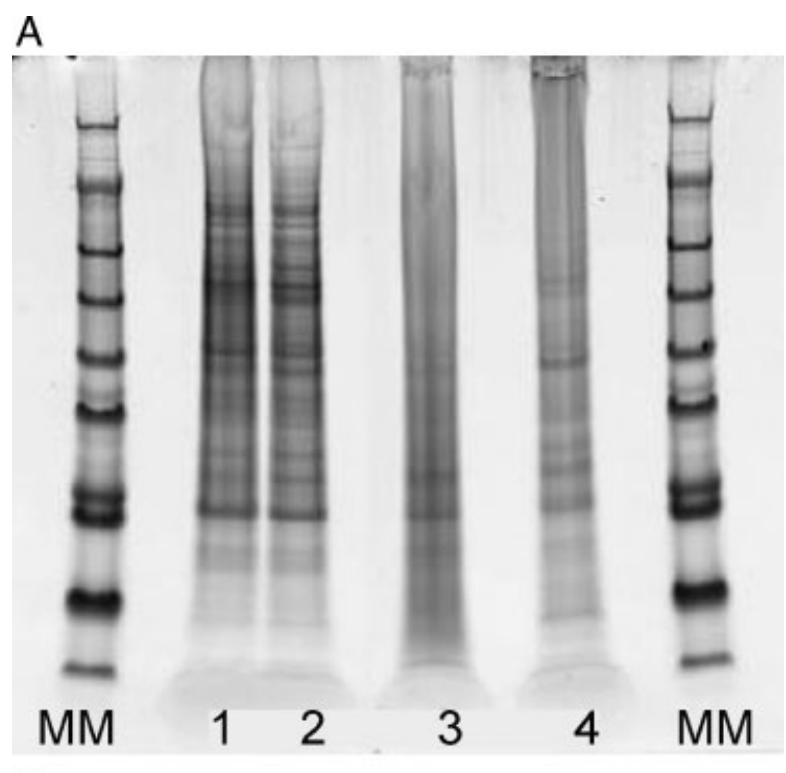

B

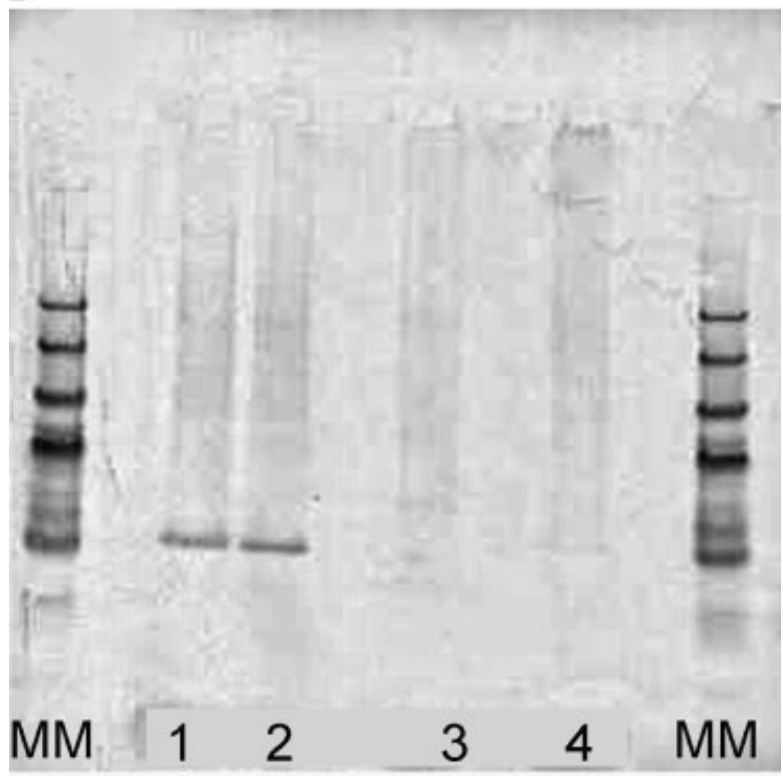

Figure 5. SDS PAGE (A) and Western blot to Api $\mathrm{g} 1$ (B) of treated samples; 1 - treatment $A 1 ; 2$ - treatment $F ; 3$-treatment $G 1$; 4 - treatment E1. Molecular weight markers are labeled MM. 

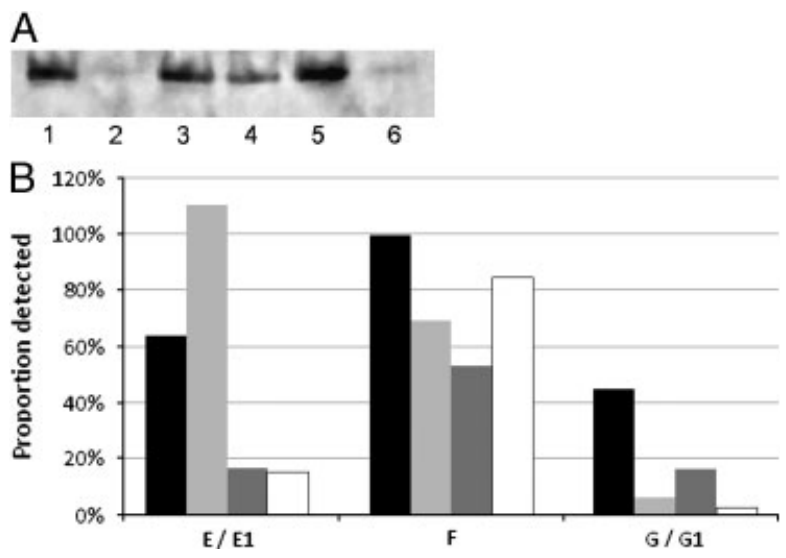

Figure 6. (A) Western blot using anti-Mal d $1 \mathrm{IgE}$ from pooled sera to detect the following: 1 - control apple, 2 - apple treatment $E, 3$ - control apple, 4 - apple treatment $F, 5$ - control apple, 6 - apple treatment G. (B) Density of bands relative to the corresponding control bands detecting Mal d 3 (light gray), Mal d $1 \mathrm{lgG}$ (black) and Mal d $1 \mathrm{lgE}$ (dark gray) and Api g $1 \mathrm{lgG}$ (white).

the concentration of PPO in apple can vary widely between varieties [27].

The effect of the matrix on Mal d 1 suggests that thermal or HP/T processing is only likely to have further decreased the amount of immunoreactive Mal d 1. This proposition is supported by the Western blots, where both the Bet $\mathrm{v} 1$ reactive IgG and the human Mal d 1 reactive IgE show similar trends. In both cases, the HP treatment alone at an initial temperature of $20^{\circ} \mathrm{C}$ had the least effect on Mal d 1 or indeed Api g 1 immunoreactivity. Figure 2 shows a loss of Mal d 1 reactivity of $75 \%$ after $60 \mathrm{~min}$ at $20^{\circ} \mathrm{C}$. The loss of Mal d 1 reactivity in the HP experiments (Figs. 3 and 6) is likely to have been much less as treatment time was much shorter $(15 \mathrm{~min})$. Both the treatments at $115^{\circ} \mathrm{C}$ significantly decreased the immunoreactivity of both Mal d 1 and Api g 1. Previous data on the loss of structure resulting from heating Mal d 1 [19] suggest that only a small proportion of the loss in reactivity is likely to have been a result of unfolding and the rest may well have been due to enzymatic modification before the enzyme itself was inactivated by the combination of $\mathrm{HP} / \mathrm{T}$.

The results for Mal d 3 are very different and demonstrate that the protein was not chemically modified after the apple flesh was cut as Mal d 1 was, even at $50^{\circ} \mathrm{C}$. We have also shown that Mal d 3 survived in apple through thermal processing, even at the extreme of $115^{\circ} \mathrm{C}$ for $10 \mathrm{~min}$. This is surprising as previous work has shown that heating LTPs in solution to $110^{\circ} \mathrm{C}$ can cause the almost complete loss of secondary structure and immunoreactivity [19, 29]. However, the protein appears to be susceptible to denaturation due to HP treatment and Fig. 5 shows a very significant loss of immunoreactivity when treated at elevated pressure and temperature.

We have compared the results on apple processing with those on celeriac and shown that the reduction in immu- noreactivity of Api g 1 in celeriac was much less. We have speculated above on the origins of the accelerated degradation of Mal d 1 and concluded that it may be due to endogenous enzymes. However, it is not clear what aspect of the matrix offered protection to the Mal d 3. As it has previously been shown that Mal d 3 is associated with apple cell walls [26], the effect of the common cell wall component pectin and its potential protective effect on Mal d 3 during heating was also investigated. We have shown in Fig. 5 that heating Mal d 3 in the presence of pectin reduced the degree of modification of the protein. The effect appeared to be independent of pectin concentration over a range of two orders of magnitude in pectin to Mal d 3 ratio. One reason for this could be that the pectin acted as an additional oxidative target. Michel et al. [30] showed that milk proteins were less denatured by $\mathrm{HP} / \mathrm{T}$ treatment in the presence of pectin but were unable to provide an explanation. Pectin has also been shown to decrease the thermal denaturation of $\beta$-lactoglobulin and subsequent hydrolysis by pepsin in simulated digestion [31]. This effect was coupled to a reduction in immunoreactivity of the digested $\beta$-lactoglobulin but again no explanation of the protective effect was proposed. While the data presented here offer a possible explanation of the potential protective effect of pectin, further work needs to be undertaken to fully understand the mechanism.

The conclusion to be drawn from these results is that $\mathrm{HP} / \mathrm{T}$ processing is an effective method to reduce the allergenicity of both apple and celeriac but for a number of different reasons depending on the allergen. Mal d 1 will have its allergenic potential reduced by disruption of the apple cell structure either prior to or during the processing. The celeriac allergen Api g 1 will have its immunoreactivity reduced by thermal processing and the apple allergen Mal d 3 will have its immunoreactivity reduced by the combination of HP and thermal processing.

The work described in this article was funded by the European Commission through the NovelQ project; contract number FOOD-CT-2005-015710, by the BBSRC as part of the Food Structure strategic programme grant to the Institute and by a fellowship within the Postdoc Programme of the German Academic Exchange Service (DAAD). The authors thank Laurian Zuidmeer at AMC in Amsterdam for providing the anti-Bet $v 1$ antibodies.

The authors have declared no conflict of interest.

\section{References}

[1] Houska, M., Strohalm, J., Kocurova, K., Totusek, J. et al., High pressure and foods - fruit/vegetable juices. J. Food Eng. 2006, 77, 386-398.

[2] Vachon, J. F., Kheadr, E. E., Giasson, J., Paquin, P., Fliss, I., Inactivation of foodborne pathogens in milk using dynamic high pressure. J. Food Prot. 2002, 65, 345-352. 
[3] San Martin, M. F., Barbosa-Canovas, G. V., Swanson, B. G., Food processing by high hydrostatic pressure. Crit. Rev. Food Sci. Nutr. 2002, 42, 627-645.

[4] Balci, A. T., Wilbey, R. A., High pressure processing of milkthe first 100 years in the development of a new technology. Int. J. Dairy Technol. 1999, 52, 149-155.

[5] De Roeck, A., Duvetter, T., Fraeye, I., Van der Plancken, L. et al., Effect of high-pressure/high-temperature processing on chemical pectin conversions in relation to fruit and vegetable texture. Food Chem. 2009, 115, 207-213.

[6] Dickinson, E., Pawlowsky, K., Effect of high-pressure treatment of protein on the rheology of flocculated emulsions containing protein and polysaccharide. J. Agric. Food Chem. 1996, 44, 2992-3000.

[7] Pfister, M. K. H., Dehne, L. I., High pressure processing - an overview of chemical changes in food. Deutsche Lebensmittel-Rundschau 2001, 97, 257-268.

[8] Sasagawa, A., Yamazaki, A., in: Hayashi, R. (Ed.), Trends in High Pressure Bioscience and Biotechnology, Proceedings, Elsevier Science Bv, Amsterdam 2002, pp. 375-384.

[9] Grauwet, T., Van der Plancken, I., Vervoort, L., Hendrickx, M. E., Van Loey, A., Protein-based indicator system for detection of temperature differences in high pressure high temperature processing. Food Res. Int. 2010, 43, 862-871.

[10] Van Eylen, D., Oey, l., Hendrickx, M., Van Loey, A., Behavior of mustard seed (Sinapis alba L.) myrosinase during temperature/pressure treatments: a case study on enzyme activity and stability. Eur. Food Res. Technol. 2008, 226, 545-553.

[11] Verlinde, P., Oey, I., Deborggraeve, W., Hendrickx, M., Loey, A., Mechanism and related kinetics of 5-methyltetrahydrofolic acid degradation during combined high hydrostatic pressure-thermal treatments. J. Agric. Food Chem. 2009, 57, 6803-6814.

[12] Meyer-Pittroff, R., Behrendt, H., Ring, J., Specific immunomodulation and therapy by means of high pressure treated allergens. High Pressure Res. 2007, 27, 63-67.

[13] Yamamoto, S., Mikami, N., Matsuno, M., Hara, T. et al., Effects of a high-pressure treatment on bovine gamma globulin and its reduction in allergenicity. Biosci. Biotechnol. Biochem. 2010, 74, 525-530.

[14] Lopez-Exposito, I., Chicon, R., Belloque, J., Recio, I. et al., Changes in the ovalbumin proteolysis profile by high pressure and its effect on IgG and IgE binding. J. Agric. Food Chem. 2008, 56, 11809-11816.

[15] Asero, R., Mistrello, G., Roncarolo, D., Amato, S., Falagiani, P., Analysis of the heat stability of lipid transfer protein from apple. J. Allergy Clin. Immunol. 2003, 112, 1009-1011.

[16] Jenkins, J. A., Griffiths-Jones, S., Shewry, P. R., Breiteneder, H., Mills, E. N. C., Structural relatedness of plant food allergens with specific reference to cross-reactive allergens: An in silico analysis. J. Allergy Clin. Immunol. 2005, 115, 163-170.
[17] Fernandez-Rivas, M., Cross-reacting allergens at the molecular scaled a north-south comparison. Revue Francaise $D$ Allergologie et D Immunol Clin 2006, 46, 167-169.

[18] Beuning, L. L., Bowen, J. H., Persson, H. A., Barraclough, D. et al., Characterisation of Mal d 1-related genes in Malus. Plant Mol. Biol. 2004, 55, 369-388.

[19] Johnson, P., Van der Plancken, I., Balasa, A., Husband, F. A. et al., High pressure, thermal and pulsed electric field induced structural changes in selected food allergens. Mol. Nutr. Food Res. 2010, 54, 1701-1710.

[20] Jankiewicz, A., Baltes, W., Bogl, K. W., Dehne, L. I. et al., Influence of food processing on the immunochemical stability of celery allergens. J. Sci. Food Agric. 1997, 75, 359-370.

[21] Ballmer-Weber, B. K., Hoffmann, A., Wuthrich, B., Luttkopf, D. et al., Influence of food processing on the allergenicity of celery: DBPCFC with celery spice and cooked celery in patients with celery allergy. Allergy 2002, 57, 228-235.

[22] Bohle, B., Zwolfer, B., Heratizadeh, A., Jahn-Schmid, B. et al., Cooking birch pollen-related food: divergent consequences for IgE- and T cell-mediated reactivity in vitro and in vivo. J. Allergy Clin. Immunol. 2006, 118, 242-249.

[23] Sancho, A. I., Rigby, N. M., Zuidmeer, L., Asero, R. et al., The effect of thermal processing on the IgE reactivity of the nonspecific lipid transfer protein from apple, Mal d 3. Allergy 2005, 60, 1262-1268.

[24] Zuidmeer, L., van Leeuwen, W. A., Budde, I. K., Breiteneder, $\mathrm{H}$. et al., Allergenicity assessment of apple cultivars: Hurdles in quantifying labile fruit allergens. Int. Arch. Allergy Immunol. 2006, 141, 230-240.

[25] Marzban, G., Puehringer, H., Dey, R., Brynda, S. et al., Localisation and distribution of the major allergens in apple fruits. Plant Sci. 2005, 169, 387-394.

[26] Borges, J. P., Jauneau, A., Brule, C., Culerrier, R. et al., The lipid transfer proteins (LTP) essentially concentrate in the skin of Rosaceae fruits as cell surface exposed allergens. Plant Physiol. Biochem. 2006, 44, 535-542.

[27] Garcia, A., Wichers, J. H., Wichers, H. J., Decrease of the IgE-binding by Mal d 1, the major apple allergen, by means of polyphenol oxidase and peroxidase treatments. Food Chem. 2007, 103, 94-100.

[28] Setinova, I., Trnkova, B., Honzova, S., Kvacova, A. et al., The influence of oxidative and polymerisation processes of apple juice on allergenicity of protein Mal d 1 in apple juice. Allergy (Oxford) 2009, 64, 244-224.

[29] Lindorff-Larsen, K., Winther, J. R., Surprisingly high stability of barley lipid transfer protein, LTP1, towards denaturant, heat and proteases. FEBS Lett. 2001, 488, 145-148.

[30] Michel, M., Leser, M. E., Syrbe, A., Clerc, M. F. et al., Pressure effects on whey protein-pectin mixtures. LWT. Food Sci. Technol. 2001, 34, 41-52.

[31] Peyron, S., Mouecoucou, J., Fremont, S., Sanchez, C., Gontard, N., Effects of heat treatment and pectin addition on beta-lactoglobulin allergenicity. J. Agric. Food Chem. 2006, 54, 5643-5650. 